\title{
BMJ Open Body image and antiretroviral therapy adherence among people living with HIV: a protocol for a systematic review and meta-analysis
}

\author{
Patrick Nyamaruze (D) , ${ }^{1}$ Richard Gregory Cowden, ${ }^{2}$ R Noah Padgett, ${ }^{3}$ \\ Kaymarlin Govender ${ }^{4}$
}

To cite: Nyamaruze $P$, Cowden RG, Padgett RN, et al. Body image and antiretroviral therapy adherence among people living with HIV: a protocol for a systematic review and meta-analysis. BMJ Open 2021;11:e045700. doi:10.1136/ bmjopen-2020-045700

- Prepublication history and additional online supplemental material for this paper are available online. To view these files, please visit the journal online. To view these files, please visit the journal online (http://dx.doi.org/10.1136/ bmjopen-2020-045700).

Received 10 0ctober 2020 Accepted 18 June 2021

\section{Check for updates}

(c) Author(s) (or their employer(s)) 2021. Re-use permitted under CC BY-NC. No commercial re-use. See rights and permissions. Published by BMJ.

${ }^{1}$ Psychology, University of KwaZulu-Natal College of Humanities, Durban, Kwazulu Natal, South Africa

${ }^{2}$ Human Flourishing Program, Institute for Quantitative Social Science, Harvard University, Cambridge, Massachusetts, USA

${ }^{3}$ Department of Educational

Psychology, Baylor University, Waco, Texas, USA

${ }^{4}$ Health Economics and HIV and AIDS Research Division, University of KwaZulu Natal, Durban, KwaZulu-Natal, South Africa

Correspondence to Mr Patrick Nyamaruze; nyamruzepatrick@yahoo.com

\section{ABSTRACT}

Introduction Adherence to antiretroviral therapy (ART) remains a key challenge to achieving the fast-track goal of ending the HIV epidemic by 2030. To provide a more comprehensive indication of whether interventions designed to promote ART adherence might benefit from targeting body image perceptions, we aim to conduct a systematic review to synthesise existing evidence on the association between body image and ART adherence. Methods and analysis A systematic review of peerreviewed observational studies and randomised controlled trials that have investigated the association between body image and adherence to ART will be performed. JSTOR, PsycARTICLES, PsycINF0, PubMed, ScienceDirect and Web of Science databases will be searched from 1 January 2000 to 31 March 2021. Eligible records will consider body image as either an independent variable or a mediator, whereas ART adherence will be assessed as an outcome variable. Study selection will follow the Preferred Reporting Items for Systematic Reviews and Meta-Analyses guidelines and study quality will be assessed using relevant tools developed by the National Institute of Health. If sufficient data are available, a meta-analysis will be conducted. Effect size estimates will be aggregated using a random effects meta-analysis approach. Publication bias and its impact will be evaluated through the use of a funnel plot and the trim-and-fill method. The Grading of Recommendations Assessment, Development and Evaluation approach will be used to report on the overall quality of evidence.

Ethics and dissemination Ethical approval is not required for a systematic review protocol. Findings of the proposed systematic review will be disseminated through conference presentations and publication in a peerreviewed journal.

PROSPERO registration number CRD42020212597.

\section{INTRODUCTION}

Approximately 24 million HIV-infected individuals are accessing antiretroviral therapy (ART). ${ }^{1}$ Early studies reported that effective ART treatment and optimal health outcomes for people living with HIV (PLHIV) requires treatment adherence that exceeds $95 \% .^{23}$ More recent evidence suggests that
Strengths and limitations of this study

- This systematic review protocol is guided by the Preferred Reporting Items for Systematic Reviews and Meta-Analyses Protocols methodology.

- The proposed systematic review will be the first to critically evaluate the quality of existing evidence from observational studies and randomised controlled trials on the association of body image with antiretroviral therapy adherence.

- Evidence synthesised in the proposed systematic review will provide insight into whether body image could offer a potentially useful avenue for promoting antiretroviral therapy adherence.

- Findings of the proposed systematic review may be limited by publication bias, study heterogeneity, the instruments that have been used to measure body image and antiretroviral therapy adherence, and the methodological quality of existing research.

viral suppression may still be achieved with less than $95 \%$ adherence levels, depending on the ART regimen, duration of treatment and previous ART use. ${ }^{45}$ However, adherence to ART remains a key challenge for PLHIV to achieve optimal health outcomes and viral suppression. $^{6}$ Poor adherence to ART can accelerate HIV resistance to treatment, ${ }^{7}$ is associated with an increased risk of HIV transmission to non-infected sexual partners, ${ }^{8}$ may have downstream implications for healthcare $\operatorname{costs}^{9}$ and is a common cause of illness and death among PLHIV. ${ }^{7}$ Common contributors to ART non-adherence are diverse. Broadly, they consist of patient-related factors, medication characteristics, health system characteristics and disease characteristics. ${ }^{10}{ }^{11}$ Experience of side effects is one commonly identified medication-related barrier to ART adherence. $^{12}$

ART has evolved over time, with newer and more effective regimens being introduced in recent years. Integrase strand transfer 
inhibitors have now become a central part of first-line treatment regimens, mainly because they tend to be more effective at reducing viral load and usually have fewer side effects compared with other treatment regimens (eg, nucleoside reverse transcriptase inhibitors) ${ }^{13}{ }^{14}$ However, the effects of integrase strand transfer inhibitor regimens on bodily changes can vary by type regimen. For example, recent findings suggest that dolutegravir may be associated with weight gain ${ }^{15}$ and raltegravir has been linked with a higher likelihood of skin rash. ${ }^{16}$

PLHIV may experience bodily changes related to HIV infection. ${ }^{17}$ Changes in body shape and composition may affect a person's body image. ${ }^{18}$ In one study conducted among PLHIV in Brazil, most participants (79.5\%) reported perceived bodily changes. ${ }^{19}$ Self-perception of body image may have important implications for the ART adherence decisions and behaviours of PLHIV. There is a growing body of evidence on the role of body image in ART adherence among PLHIV. For example, negative body image is associated with ART non-adherence and loss of retention in care, ${ }^{20}$ and body image disturbance has been linked to ART adherence problems in homosexual men. ${ }^{21}$ One systematic review evaluating the effect of adverse antiretroviral drug reactions on ART adherence found that visually noticeable adverse drug reactions were more likely to disrupt adherence compared with other adverse drug reactions. ${ }^{22}$ In addition to its exclusive focus on qualitative evidence that previous review did not focus specifically on body image.

Body image generally refers to the perception that a person has of their body. ${ }^{23}$ It is composed of several dimensions, including appearance, functioning of the body and physical competence. ${ }^{24}$ Body image perceptions are influenced by many factors (eg, peers, age, gender, culture). For example, young people tend to have higher levels of body image disturbance than older people. ${ }^{25}$ Some evidence suggests that females may have lower levels of body image satisfaction than males. ${ }^{26}$

Challenges involving the conceptualisation of body image are reflected in the varied ways the concept has been applied when investigating ART adherence. Specifically, body image has been equated to body dissatisfaction, ${ }^{21}$ body image disturbance,${ }^{27}$ body image perception ${ }^{28}$ and body weight. ${ }^{29}$ Similarly, different approaches have been used to measure body image, including the Body Change and Distress Questionnaire-Short Form, ${ }^{30}$ the use of a select set of items from an HIV Symptom Index, ${ }^{31}$ and a figure rating scale composed of a set of silhouettes representing a continuum of body shapes ranging from thinness to obesity. ${ }^{32}$ Some of these existing measurement approaches may not adequately capture the concept of body image. For example, the figure rating scale calculates a person's body image score as the difference between the ideal and the real silhouette. This approach may be useful for identifying body image perceptions held by a person, but it is unlikely to uncover their feelings (negative/positive) about their body. In light of the variety of ways that body image has been measured, one purpose of the proposed review is to synthesise the approaches that have been used to assess body image in research on ART adherence.

As the roll-out of ART continues, issues related to body image and its impact on ART adherence are becoming increasingly important. Some evidence suggests that Cognitive-Behavioural Therapy for Body Image and Self-Care (CBT-BISC) can effectively reduce body image disturbance and increase ART adherence. ${ }^{2733}$ One of the ways in which CBT-BISC positively impacts ART adherence is through reductions in body image disturbance. Although attention has been directed to systematic reviews focused on identifying barriers experienced by PLHIV when accessing ART, ${ }^{34} 35$ a systematic review on the relation between body image and ART adherence has yet to be conducted. To address this issue, we will perform a systematic review of existing evidence from observational studies and randomised controlled trials (RCTs) on the association of body image with ART adherence. If more than three records meeting the criteria for inclusion are identified, the systematic review will be accompanied by a meta-analysis. ${ }^{36}$ The main purpose of the proposed systematic review is to examine the association between body image and ART adherence by providing a meaningful synthesis of the extant literature. Specifically, two research questions will guide the proposed systematic review: (1) how has body image been conceptualised and measured in research involving ART adherence? and (2) is positive body image associated with higher levels of ART adherence among PLHIV? By synthesising available evidence on the conceptualisation and measurement of body image and its association with ART adherence, the findings of the proposed review will provide evidence that could be used to inform the design of standalone or multipronged interventions that seek to promote ART adherence by targeting body image.

\section{METHODS AND ANALYSIS \\ Study design}

The steps of the proposed systematic review will include outlining the search strategy, the inclusion and exclusion criteria, finding studies, selecting those studies that address the review question and meet the inclusion criteria, and extracting and synthesising data. This systematic review protocol is guided by the Preferred Reporting Items for Systematic Reviews and Meta-analyses (PRISMA) Protocol checklist (online supplemental file 1). ${ }^{37}$

\section{Search strategy}

We will perform an electronic database search in JSTOR, PsycARTICLES, PsycINFO, PubMed, ScienceDirect and Web of Science to identify an initial set of records. A twopart search strategy will be used to identify studies that are eligible for inclusion. First, we will search electronic bibliographic databases for published studies using a string of preselected key words and phrases. Second, we will search the reference lists of records that meet the 
criteria for inclusion in the review and the reference lists of relevant, previously published review articles for other records that may be eligible for inclusion.

\section{Inclusion and exclusion criteria}

The study population will include PLHIV and who are on ART treatment. There will be no limits on study participants in terms of age, gender and ethnicity. The selection of studies will be restricted to RCTs and observational studies involving cross-sectional, case-control and longitudinal designs. Studies that use either validated and non-validated measures of one or more dimensions of body image and ART adherence will be included, regardless of the measurement approach (eg, self-report, report cards). We will exclude studies that examine lipodystrophy as an independent variable, because it is a factor that may contribute to a negative body image rather than a dimension or component of body image itself. ${ }^{32}{ }^{38}$ However, if there are sufficient studies that both meet the inclusion criteria and report on lipodystrophy, we will include lipodystrophy as a covariate and report the percentage of participants who have lipodystrophy. Eligible records will consider body image as either an independent variable or a mediator, while ART adherence will be assessed as a criterion or outcome variable. Due to the ambiguity of parsing out effects of specific treatment components when interventions are multifaceted, only RCTs that exclusively and explicitly target body image or examine body image as a mechanism (ie, mediator) by which the treatment is associated with ART adherence will be included. We will include studies that have been peer-reviewed and published. We will exclude papers that are not available in English. Moreover, qualitative studies, mixed-method studies, commentaries, study protocols, literature reviews, conceptual papers and conference abstracts will also be excluded. We will manually check the reference lists of included articles and relevant review papers to identify additional studies that were not revealed during the formal database search. The Rayyan QCRI (https://rayyan.qcri.org) cloud-based platform will be used to manage the review process from initial screening of records through to selection of studies that are eligible for inclusion. ${ }^{39}$

\section{Time period}

All eligible studies published from 1 January 2000 to 31 March 2021 will be included. The period from early 2000 to the present marks an era of large-scale ART coverage, classifiable as (1) ART introduction 2000-2007, (2) expanded ART (2008-2010) and (3) scaled-up ART (2011-present). The progression in ART availability and usage during this time frame offers a suitable context for documenting the possible implications of long-term use of ART for PLHIV, such as the decline in healthy body image and its impact on ART adherence.

\section{Study screening and selection}

The first review author will use a set of keywords to search databases to identify studies that potentially meet the inclusion criteria outlined above. The search words for body image were informed by current literature and by Thompson $e t a t^{t 0}$ who identified a range of terms that either referred to body image or a subdimension of body image. The search terms will be combined using the Boolean operators 'AND' and 'OR'. The preliminary search strategy that was developed is detailed in table 1 . The search strategy will be adjusted to fit the syntax specifications of each electronic database. Given the iterative nature of systematic reviews and the potential for additional search terms to be discovered during the search process, final search queries in each database will

\begin{tabular}{|c|c|}
\hline Databases & Search items \\
\hline \multirow{4}{*}{$\begin{array}{l}\text { JSTOR } \\
\text { PsycARTICLES } \\
\text { PsycINFO } \\
\text { PubMed } \\
\text { ScienceDirect } \\
\text { Web of Science }\end{array}$} & $\begin{array}{l}\text { \#1: (("body image" (Title/Abstract) OR "body dissatisfaction" (Title/Abstract) OR "body image disturbance" } \\
\text { (Title/Abstract) OR "body concern"” (Title/Abstract) OR "body satisfaction" (Title/Abstract) OR "body } \\
\text { dysphoria" (Title/Abstract) OR "appearance evaluation" (Title/Abstract))) }\end{array}$ \\
\hline & $\begin{array}{l}\text { \#2: (("antiretroviral therapy adherence" (Title/Abstract) OR "antiretroviral therapy non-adherence" } \\
\text { (Title/Abstract) OR "antiretroviral therapy nonadherence" (Title/Abstract) OR "antiretroviral treatment } \\
\text { adherence" (Title/Abstract) OR "antiretroviral treatment non-adherence" (Title/Abstract) OR "antiretroviral } \\
\text { treatment nonadherence" (Title/Abstract) OR "antiretroviral therapy uptake adherence" (Title/Abstract) } \\
\text { OR "antiretroviral therapy uptake non-adherence" (Title/Abstract) OR "antiretroviral therapy uptake } \\
\text { nonadherence" (Title/Abstract) OR "ART adherence" (Title/Abstract) OR "ART non-adherence" (Title/ } \\
\text { Abstract) OR "ART nonadherence" (Title/Abstract) OR "nucleoside reverse transcriptase inhibitor" (Title/ } \\
\text { Abstract) OR "nucleotide reverse transcriptase inhibitor" (Title/Abstract) OR "non-nucleoside reverse } \\
\text { transcriptase inhibitor" (Title/Abstract) OR "nonnucleoside reverse transcriptase inhibitor" (Title/Abstract) } \\
\text { OR "protease inhibitor*" (Title/Abstract) OR "integrase inhibitor*" (Title/Abstract) OR "entry inhibitor*" (Title/ } \\
\text { Abstract))) }\end{array}$ \\
\hline & \#3: ((“HIV” (Title/Abstract) OR “AIDS” (Title/Abstract) OR “HIV/AIDS” (Title/Abstract))) \\
\hline & \#4: \#1 AND \#2 AND \#3 \\
\hline
\end{tabular}

JSTOR, Journal Storage; PsycINFO, Psychology Information. 
be published alongside the systematic review as online supplemental file 2.

\section{Data extraction}

When feasible, we will restrict the screening process to the title and abstract. However, if all the keywords necessary to include the record are not present in the abstract or title, the first review author will screen the entire article to decide whether or not it should be included. References of records that meet the criteria for inclusion will be searched manually to identify further eligible studies. The first and second review authors will extract the relevant data from eligible records and independently assess each for eligibility using a data extraction form that will be developed to standardise the data extraction process. The third review author will arbitrate any disagreements between the two review authors over the eligibility of particular records. A PRISMA flow diagram will be prepared to summarise the record screening and assessment process.

\section{Data items}

The authors will extract the following information from each of the included studies: (1) general characteristics of the publication: title, year of publication, first author last name and purpose of the study; (2) characteristics of the participants: age, sex, weight gain, mode of HIV transmission (ie, vertical vs horizontal), ART regimen, concurrent medication use and sexual orientation of participants; (3) research strategy: overall research design, number of participants, and country where participants were sampled from; (4) instruments: type of instruments used to measure body image and ART adherence, dimensionality of measures and method of data collection (eg, selfadministered, interviewer administered); instrument reliability; (5) analysis and results: primary statistical analysis, variables included in the analyses and main outcomes (including potential confounding factors that were taken into consideration). If any included studies report results for multiple groups independently, we will (A) specify the groups used to subset the results, (B) report the overall effect size and (C) report descriptive statistics for relevant groups. We will contact the authors of included studies publications for missing outcome data or unclear information.

\section{Data management}

We will implement the search strategies and import all references identified into EndNote. We will record and report details on the number of full text papers obtained and the number of included and excluded articles. The search results from the different electronic databases will be combined in a single EndNote library and duplicate records will be removed.

\section{Risk of bias in individual studies}

The quality of the identified studies will be assessed independently by two reviewers using the Quality Assessment of Controlled Intervention Studies, the Quality
Assessment Tool for Observational Cohort and CrossSectional Studies, and the Quality Assessment of CaseControl Studies tool provided by the National Institute of Health (2015). ${ }^{41}$ Studies will be rated as good, fair and poor quality, where high risk of bias is translated to a rating of poor quality ('-') and low risk of bias is translated to a rating of good quality ('+'). A traffic light plot will be generated to illustrate the proportion of studies classified as good, fair and poor, both for each category and overall.

\section{Data synthesis}

Effects from records included in the proposed review and meta-analysis will be coded to reflect the relation between positive body image and ART adherence. To aggregate the results across the studies identified, we will first convert all study results to a common effect size metric (ie, correlation coefficient). Fisher transformations will be used to convert correlations to approximate z-scores, which helps to stabilise the variance. ${ }^{42}$ The estimated sampling error of each correlation is approximated using the SE resulting from the Fisher transformation method. Given a sample correlation $r_{i}$ in study $i$ with study sample size, the second order approximations ${ }^{43}$ to the corresponding $z_{i}$ are

$$
\begin{gathered}
z_{i}=\operatorname{arctanh}\left(r_{i}\right)+\frac{r_{i}}{2 n_{i}}=\frac{1}{2}\left(\frac{1+r_{i}}{1-r_{i}}\right)+\frac{r_{i}}{2 n_{i}} \\
\operatorname{VAR}\left(z_{i}\right)=\frac{1}{n_{i}}+\frac{6-r_{i}^{2}}{2 n_{i}^{2}} \\
r_{i}=\frac{\ln \left(O R_{i}\right)}{\sqrt{\ln \left(O R_{i}\right)^{2}+\frac{2.89 n^{2}}{n_{1} n_{2}}}}
\end{gathered}
$$

Some studies may conduct logistic regression using ART adherence as a dichotomous criterion or outcome variable. In such cases, we will use the resulting betacoefficients and the corresponding log OR to compute the $r_{i}$ between positive body image and ART adherence. For a given odds ratio $\left(O R_{i}\right)$, we will use the Ulrich and Wirtz approximation to transformation ORs to correlations, ${ }^{44}$ that is

where with $n_{1}$ and $n_{2}$ being the number of subjects in each adherence group. We will then use this in (1) and (2) to transform the converted correlation to a z-score. Lastly, some studies may report the association between negative body image and ART non-adherence. In such cases, we will reverse the sign so that scores correspond to the association between positive body image and ART adherence. If additional unforeseen transformations are needed, we will make note and describe how these additional transformations were conducted.

The effect size estimates will be aggregated using a random effects meta-analysis approach. We will conduct a linear mixed-effects model with normally distributed errors. The within-study variation will be captured by the approximate sampling variance given by Equation (2). 
An unconditional model and a full conditional model will be considered for analysis. The unconditional model will be a random effects only model. For this, we mean that only the average effect size and the heterogeneity in effect sizes will be estimated. For the full conditional model, we intend to explain potential sources for why studies may differ in effect sizes. The size of the full model will depend on the final number of studies eligible for analysis (see inclusion criteria). If between three and five studies are included in the analysis, we will restrict our model to only approximate the average effect size, the heterogeneity of effect sizes, and one covariate (eg, research design, gender). If more than five studies are extracted, we will expand the full model to include additional characteristics. For every three additional studies an additional design factor will be included. For example, with six studies, two study characteristics will be included; with nine studies, three characteristics; and with 12 studies, four characteristics. The specific characteristics included in the full model will be determined by what characteristics are available, substantive importance and magnitude of effect. The effects of each design factor will be estimated as fixed across studies. The full random effects model can be expressed as

Where,

$$
z_{i}=\theta+\sum_{\{p=1\}}^{P} \gamma_{p} X_{p}+u_{i}
$$

$z_{i}$ is the normally distributed sample z-transformed correlation where $z_{i}$ has known variance, that is $z_{i} \sim N\left(\mu_{z_{i}}, \sigma_{i}^{2}\right)$. Each study may have its own subpopulation but the overall distribution of $z_{i}$ across studies is the main focus of this meta-analysis. That is, each $\mu_{z_{i}}$ comes from a population with mean $\theta$,

$\theta$ is the population effect-size,

$X_{p}$ is the $p^{t h}$ study characteristic (ie, covariate) included in the study where $\gamma_{p}$ is the estimated differences in the population effect size relative to changes in the covariate. All $X_{p}$ 's will be mean centred such that the $\theta$ does not change meaning,

oFor example, if $X_{p}$ represents the proportion of females in the study, we would mean centre each estimate relative the average proportion of females across all studies, $\mu_{i}$ is the deviation score associated with each study. The deviations are assumed to be normally distributed, that is $u_{i} \sim N\left(0, \tau^{2}\right)$. An important result is the variance of $u_{i}$. That is, $\operatorname{VAR}\left(u_{i}\right)=\tau^{2}$ represents the between-study heterogeneity.

This relationship results in a linear hierarchical model where each effect size is drawn from a population, which we can restate the model without the deviation scores $u_{i}$ by using $\mu_{z_{i}} \sim N\left(\theta, \tau^{2}\right)$. The more elaborate approach makes it easier to see the deviation between the overall estimate of effect size $(\theta)$ and the study estimates.

Models will be estimated using a Bayesian framework. ${ }^{45}$ Estimation will be carried out with Stan, ${ }^{46}$ using the rstan package $^{47}$ in $\mathrm{R}$. The prior specification depends on the number of studies included in the meta-analysis. Generally, the prior choice for the fixed effects make little difference in the estimation of a random effects model. The priors are the variance components and tend to be highly influential, especially when the number of studies is below five. ${ }^{45} 48$ This is the statistical reason why only one fixed effect is to be included in the full model when the number of studies is five or fewer. We will use multiple sets of priors for each model to investigate the sensitivity of the meta-analysis results to selection of prior.

For the unconditional model, the full prior specifications are shown in figure 1 , online supplemental file 3 . For the relatively uninformative prior structure detailed in figure 1 (Panel A), all priors were selected to be flat (or essentially flat) over a logical range of possible values for $\theta$ and $\tau$. The upper bound of two was chosen for the uniform prior on $\tau$ because each was mapped to a standard normal distribution with SD of 1 . This means that the SD of the estimated effect sizes is most likely less than 1 , but using a uniform prior with a relatively high upper bound of 2 allows for all plausible values with equal likelihood. However, the uniform prior for the SD can lead to potential bias in the estimates, as the use of the uniform prior has been shown to overestimate effects if the upper bound is too high. ${ }^{48}$ The relatively informative prior structure is shown in figure 1 (Panel B). The prior $\theta$ was chosen to be more informative of our belief that the association
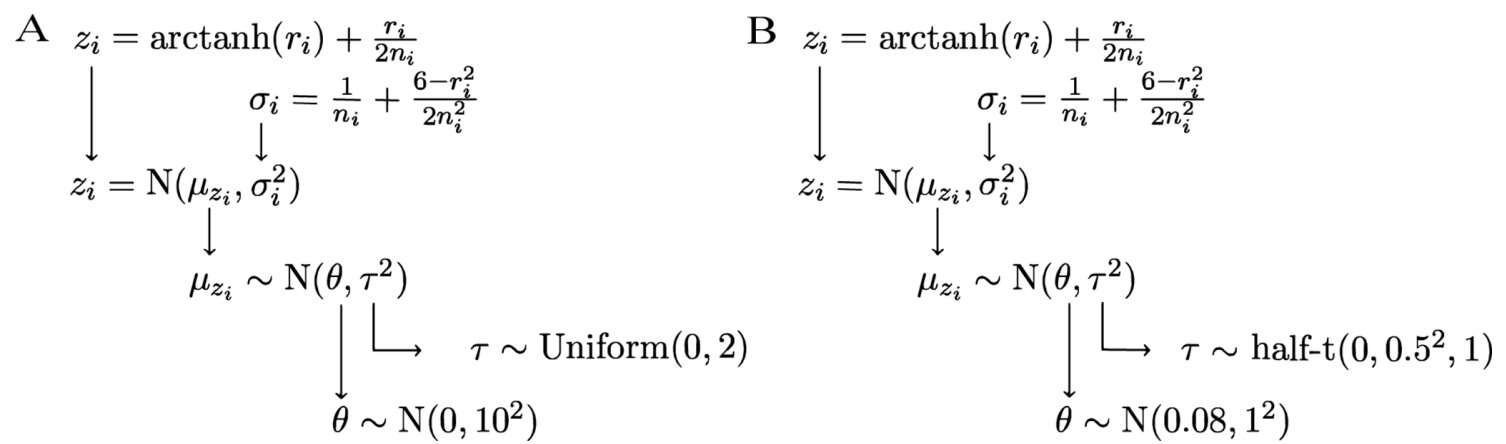

Figure 1 Prior specifications for unconditional random effects model. (A) The prior structure is relatively non-informative with diffuse priors for the population mean effect size $(\theta)$ and between-study heterogeneity $(\tau)$ parameters; (B) prior structure is relatively informative with lower variance priors for the population mean effect size and between-study heterogeneity parameters. Similar in both models is the transformation of the observed correlations $\left(r_{i}\right)$ and study sample sizes $\left(n_{i}\right)$ into the effect sizes $\left(z_{i}\right)$ and study variance estimates $\left(\sigma_{i}\right)$. 


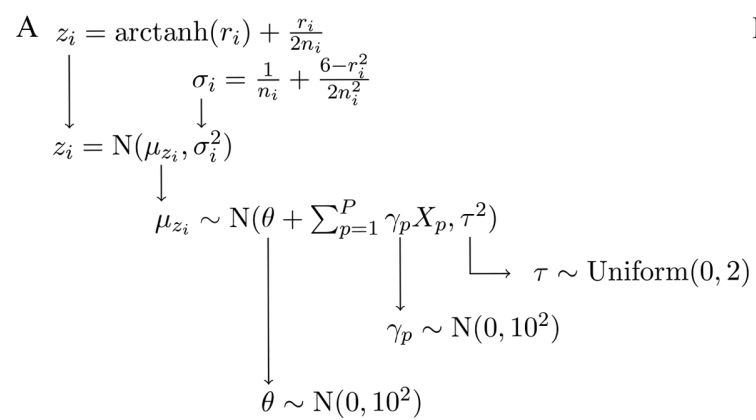

$$
\begin{aligned}
& \text { B } z_{i}=\operatorname{arctanh}\left(r_{i}\right)+\frac{r_{i}}{2 n_{i}}
\end{aligned}
$$

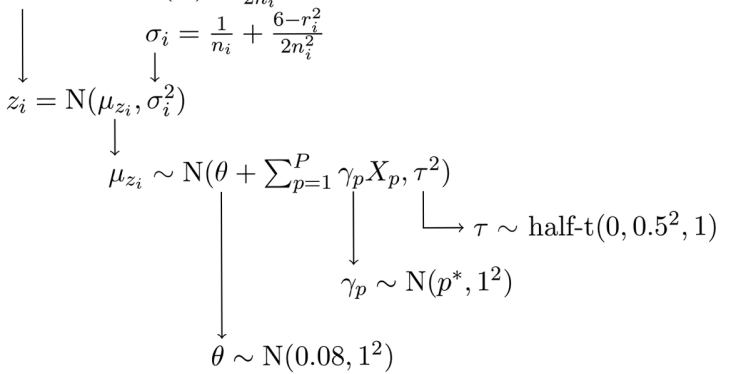

Figure 2 Prior specifications for full conditional random effects model. (A) The prior structure is relatively non-informative with diffuse priors for the population mean effect size, $(\theta$ between-study heterogeneity, and regression weight of study design characteristic $\left(\gamma_{p}\right)$ parameters; (B) Prior structure is relatively informative with lower variance priors for the population mean effect size, between-study heterogeneity and regression weight of study design characteristic parameters. Similar in both models is the transformation of the observed correlations $\left(r_{i}\right)$ and study sample sizes $\left(n_{i}\right)$ into the effect sizes $\left(z_{i}\right)$ and study variance estimates $(\tau)$.

between positive body image and ART adherence is positive. This means that previous research has shown that the association is positive. ${ }^{31}$ The centre of the normal prior was chosen to be the Fisher transformation of the average of reported correlations $\left(r=\frac{0.12+0.04}{2}=0.08\right)$ in two previous studies. ${ }^{2130}$ To provide a relatively informative comparison to the prior used in (Panel A), the SD of this prior was chosen to be one. The half-t prior for $\tau$ was chosen based on the Gelman's ${ }^{48}$ recommendations for priors when the number of studies is below five. The variance of the half-t prior was selected to 0.25 , which means that we suspect the between-study variability will be small relative the standard normal distribution. The $\mathrm{df}$ for the half-t was selected to be one.

For the full conditional model, the prior structure will be nearly identical but the priors for the regression weights $\left(\gamma_{p}\right)$ parameters were added. In figure 2, online supplemental file 4 , the priors for the regression weights are relatively diffuse to provide a relatively non-informative prior for the effect of each study characteristic. The relatively non-informative priors for each $\gamma_{p}$ were chosen to be the same as the prior for (ie, normally distributed with large variance). In figure $2 \mathrm{~B}$, the priors for the regression weights are specified to be relatively informative to match the relatively informative prior structure specified for the other model parameters. The relatively informative priors for covariate effects $\left(\gamma_{p}\right)$ will be based on content expertise from the first author and relevant a priori literature. For example, under a potential gender effect for studies with different proportions of females, we selected the regression weight to be positive $\left(p^{*}=0.10\right)$. This specification indicates that studies with more females are likely to exhibit a stronger association between positive body image and ART adherence.

A prior-sensitively analysis will be conducted for each prior structure. The sensitivity analysis will test how dependent the posterior of the variance $\tau$ is to the chosen prior. For the relatively non-informative uniform prior, this will be done by changing the upper bound to smaller and larger values. For the half-t prior, the variance will be decreased and increased. We will indicate whether the initial prior structures were influential and how we selected priors to be less influential.

After we have completed necessary assessments of the posterior distributions, we will test for evidence of a positive association between positive body image and ART adherence by computing the probability that the pooled effect size is positive. This will be done using the posterior distribution of $\theta$ from the above models. If $\theta>0$, then we have evidence that the correlation is positive. To facilitate interpretation of results, we will back transform the results to the correlation scale. We will note any potential differences in conclusions about the association between body image and ART adherence across the four models. We will use forest plots to display the results of the estimated study effect sizes and the pooled effect size estimate. If quantitative synthesis is not appropriate, the extracted studies will be described narratively to summarise and explain the characteristics and findings of the included studies.

\section{Meta-bias(es)}

To evaluate publication bias, we will follow the recommendations of Sterne $e t a t^{49}$ by graphically representing the relationship between the observed effect sizes and estimation precision using funnel plots. Ideally, the plot appears symmetrical and points on the plot form an inverted funnel. Studies with smaller sizes tend to be scattered widely at the bottom of the plot whereas studies with larger sample sizes typically have greater precision in the estimates of effect sizes and are centrally located at the top of the funnel. Asymmetrical plots or blank spots within plots are evidence of potential bias. The asymmetry of the plot will be tested using Eggar's test. ${ }^{50}$ The statistical test of asymmetry will only be conducted if a minimum number of studies (10) are included in the meta-analysis. ${ }^{51}$ Additionally, the trim-and-fill method will be applied to assess the impact of potential publication bias. $^{52}$

Another potential source of bias in our results is a lack of available information about confounding variables. One such source is that different antiretroviral 
drugs/classes of drugs are related to weight gain, which is linked to body image. Knowledge of which classes of drugs were used in a particular study may then be linked to the observed effect size of the relationship between ART adherence and body image by the impact that particular classes of drugs have on weight gain. Although weight gain and class of drug may potentially be reported in each study that meet inclusion criteria, our preliminary searches indicate that such information is not widely reported. We expect a large amount of missing data for some of these potentially confounding variables. The potential bias resulting from lack of information about confounders will be investigated using sensitivity analyses. ${ }^{53}$ Similar to how we will be conducting prior-sensitivity analyses, additional sensitivity analyses will be performed to investigate how strong the effect of an omitted confounder would need to be in order to change the substantive conclusions of the meta-analysis.

\section{Confidence in cumulative evidence}

The Grading of Recommendations Assessment, Development and Evaluation (GRADE) approach will be used to report on the overall quality of evidence for the outcome of interest. ${ }^{54}$ In brief, the two review authors will assess the quality of evidence across the domains of risk of bias, consistency, directness, precision and publication bias. Additionally, we will assess the magnitude of effect. A quality of evidence assessment presented alongside the effect estimates provides an indication of how much confidence can be placed in the findings. As a result of applying GRADE, two authors will independently rate the quality of the reviewed records and assign an overall quality rating of very low, low, moderate or high. ${ }^{55}$ These four categories reflect the level of confidence the reviewers have in the quality of the evidence. The high category indicates that further research is very unlikely to change the level of confidence in the estimated effect, whereas a very low category indicates uncertainty about the estimated effect.

\section{CONCLUSION}

ART adherence is crucial to maintaining the health and well-being of PLHIV. Evidence from both high-income and low-income countries suggests that achieving optimal ART adherence is challenging and that adherence deteriorates over time as side effects of mediation increase. ${ }^{56}$ Given the centrality of body image to the lives and ART adherence decisions of PLHIV, ${ }^{31} 57$ mechanisms need to be identified to promote optimal adherence to treatment regimens. By synthesising evidence on the relation between body image and ART adherence, the findings of the proposed systematic review could be used to inform the development of interventions that target body image as a means of improving ART adherence and promoting well-being among PLHIV.

\section{Amendments}

The protocol for this study will be amended as necessary.

Twitter Patrick Nyamaruze @Partypee1

Contributors PN and RGC conceptualised and designed the protocol, drafted the initial manuscript and reviewed the manuscript. PN, RGC and NP defined the concepts and search items, data extraction process and methodological appraisal of the studies. RGC and NP planned the data extraction and statistical analysis. KG, RGC and NP provided critical insights. All authors have approved and contributed to the final written manuscript.

Funding The authors have not declared a specific grant for this research from any funding agency in the public, commercial or not-for-profit sectors.

Competing interests None declared.

Patient and public involvement statement Patients or the public were not involved in the design, or conduct, or reporting, or dissemination plans of our research.

\section{Patient consent for publication Not required.}

Provenance and peer review Not commissioned; externally peer reviewed.

Supplemental material This content has been supplied by the author(s). It has not been vetted by BMJ Publishing Group Limited (BMJ) and may not have been peer-reviewed. Any opinions or recommendations discussed are solely those of the author(s) and are not endorsed by BMJ. BMJ disclaims all liability and responsibility arising from any reliance placed on the content. Where the content includes any translated material, BMJ does not warrant the accuracy and reliability of the translations (including but not limited to local regulations, clinical guidelines, terminology, drug names and drug dosages), and is not responsible for any error and/or omissions arising from translation and adaptation or otherwise.

Open access This is an open access article distributed in accordance with the Creative Commons Attribution Non Commercial (CC BY-NC 4.0) license, which permits others to distribute, remix, adapt, build upon this work non-commercially, and license their derivative works on different terms, provided the original work is properly cited, appropriate credit is given, any changes made indicated, and the use is non-commercial. See: http://creativecommons.org/licenses/by-nc/4.0/.

ORCID iD

Patrick Nyamaruze http://orcid.org/0000-0003-2390-5307

\section{REFERENCES}

1 UNAIDS. AIDSinfo. 2020, 2020. Available: http://aidsinfo.unaids.org/ [Accessed 20 Dec 2020].

2 Bangsberg DR, Hecht FM, Charlebois ED, et al. Adherence to protease inhibitors, HIV-1 viral load, and development of drug resistance in an indigent population. AIDS 2000;14:357-66.

3 Paterson DL, Swindells S, Mohr J, et al. Adherence to protease inhibitor therapy and outcomes in patients with HIV infection. Ann Intern Med 2000;133:21-30.

4 Lima VD, Bangsberg DR, Harrigan PR. Risk of viral failure declines with duration of suppression on HAART, irrespective of adherence level. J Acquir Immune Defic Syndr 2010;55:460-5.

5 Bangsberg DR. Less Than 95\% Adherence to Nonnucleoside Reverse-Transcriptase Inhibitor Therapy Can Lead to Viral Suppression. Clin Infect Dis 2006;43:939-41.

6 Soomro N, Fitzgerald G, Seeley J, et al. Comparison of antiretroviral therapy adherence among HIV-infected older adults with younger adults in Africa: systematic review and meta-analysis. AIDS Behav 2019;23:445-58.

7 B. Nachega J, C. Marconi V, U. van Zyl G, et al. Hiv treatment adherence, drug resistance, virologic failure: evolving concepts. Infect Disord Drug Targets 2011;11:167-74.

8 Stekler JD, Milne R, Payant R, et al. Transmission of HIV-1 drug resistance mutations within partner-pairs: a cross-sectional study of a primary HIV infection cohort. PLoS Med 2018;15:e1002537.

9 Long LC, Fox MP, Sauls C, et al. The high cost of HIV-positive inpatient care at an urban hospital in Johannesburg, South Africa. PLoS One 2016;11:e0148546.

10 Azia IN, Mukumbang FC, Van Wyk B. Barriers to adherence to antiretroviral treatment in a regional hospital in Vredenburg, Western Cape, South Africa. South Afr J HIV Med 2016;17.

11 Coetzee B, Kagee A, Vermeulen N. Structural barriers to adherence to antiretroviral therapy in a resource-constrained setting: the perspectives of health care providers. AIDS Care 2011;23:146-51. 
12 Renju J, Moshabela M, McLean E, et al. 'Side effects' are 'central effects' that challenge retention in HIV treatment programmes in six sub-Saharan African countries: a multicountry qualitative study. Sex Transm Infect 2017:93:e052971.

13 d'Arminio Monforte A, Cozzi-Lepri A, Di Biagio A, et al. Durability of first-line regimens including integrase strand transfer inhibitors (INSTIs): data from a real-life setting. J Antimicrob Chemother 2019;74:1363-7.

14 Park TE, Mohamed A, Kalabalik J, et al. Review of integrase strand transfer inhibitors for the treatment of human immunodeficiency virus infection. Expert Rev Anti Infect Ther 2015;13:1195-212.

15 Norwood J, Turner M, Bofill C, et al. Brief report: weight gain in persons with HIV switched from Efavirenz-Based to integrase strand transfer Inhibitor-Based regimens. J Acquir Immune Defic Syndr 2017;76:527-31.

16 Nachman S, Alvero C, Teppler H, et al. Safety and efficacy at 240 weeks of different raltegravir formulations in children with HIV-1: a phase 1/2 open label, non-randomised, multicentre trial. Lancet HIV 2018;5:e715-22.

17 Fingeret MC, Vidrine DJ, Arduino RC, et al. The association between body image and smoking cessation among individuals living with HIV/AIDS. Body Image 2007;4:201-6.

18 Yang HJ, Lee HK, Kim M-R. The concept analysis of body image of people living with HIV/AIDS. IJBSBT 2015;7:315-24.

19 Giudici KV, Duran ACFL, Jaime PC. Self-Reported body changes and associated factors in persons living with HIV. $J$ Health Popul Nutr 2010;28:560-6

20 Ezekiel MJ, Talle A, Juma JM, et al. "When in the body, it makes you look fat and HIV negative": The constitution of antiretroviral therapy in local discourse among youth in Kahe, Tanzania. Soc Sci Med 2009;68:957-64.

21 Blashill AJ, Vander Wal JS. The role of body image dissatisfaction and depression on HAART adherence in HIV positive men: tests of mediation models. AIDS Behav 2010;14:280-8.

$22 \mathrm{Li} \mathrm{H}$, Marley G, Ma W, et al. The role of ARV associated adverse drug reactions in influencing adherence among HIV-infected individuals: a systematic review and qualitative meta-synthesis. AIDS Behav 2017;21:341-51.

23 Grogan S. Body image: understanding body dissatisfaction in men, women and children. 2nd edn. London: Routledge, 2008.

24 Cash TF, Pruzinsky T. (2002) Future challenges for body image theory, research, and clinical practice. In: Pruzinsky T, ed. Body image: A handbook of theory, research, and clinical practice. New York, NY: Guilford Press, 2012: 509-16.

25 Bucchianeri MM, Arikian AJ, Hannan PJ, et al. Body dissatisfaction from adolescence to young adulthood: findings from a 10-year longitudinal study. Body Image 2013;10:1-7.

26 Brennan MA, Lalonde CE, Bain JL. Body image perceptions: do gender differences exist? PsiChiJournal 2010;15:130-8.

27 Lamb KM, Nogg KA, Safren SA, et al. Mechanism of change in cognitive behavioral therapy for body image and self-care on art adherence among sexual minority men living with HIV. AIDS Behav 2018:22:2711-7.

28 Plankey M, Bacchetti P, Jin C, et al. Self-Perception of Body Fat Changes and HAART Adherence in the Women's Interagency HIV Study. AIDS Behav 2009;13:53-9.

29 van Griensven J, Zachariah R, Mugabo J, et al. Weight loss after the first year of stavudine-containing antiretroviral therapy and its association with lipoatrophy, virological failure, adherence and CD4 counts at primary health care level in Kigali, Rwanda. Trans $R$ Soc Trop Med Hyg 2010;104:751-7.

30 Blashill AJ, Goshe BM, Robbins GK, et al. Body image disturbance and health behaviors among sexual minority men living with HIV. Health Psychology 2014;33:677-80.

31 Justice AC, Holmes W, Gifford AL, et al. Development and validation of a self-completed HIV symptom index. J Clin Epidemiol 2001;54 Suppl 1:S77-90.

32 Lima L, Teixeira DM, Martins PC. Body image and anthropometric indicators in adolescents living with HIV. Rev Bras Cineantropom Desempenho Hum 2018;20:53-63.
33 Blashill AJ, Safren SA, Wilhelm S, et al. Cognitive behavioral therapy for body image and self-care (CBT-BISC) in sexual minority men living with HIV: a randomized controlled trial. Health Psychology 2017;36:937-46.

34 Kanters S, Park JJH, Chan K, et al. Interventions to improve adherence to antiretroviral therapy: a systematic review and network meta-analysis. The Lancet HIV 2017;4:e31-40.

35 Shah R, Watson J, Free C. A systematic review and meta-analysis in the effectiveness of mobile phone interventions used to improve adherence to antiretroviral therapy in HIV infection. BMC Public Health 2019;19:915.

36 Valentine JC, Pigott TD, Rothstein HR. How many studies do you need? A primer on statistical power for meta-analysis. J Educ Behav Stat 2010;35:215-47.

37 Moher D, Shamseer L, Clarke M, et al. Preferred reporting items for systematic review and meta-analysis protocols (PRISMA-P) 2015 statement. Syst Rev 2015;4:1.

38 Adams C, Stears A, Savage D, et al. "We're stuck with what we've got": The impact of lipodystrophy on body image. J Clin Nurs 2018;27:1958-68.

39 Ouzzani M, Hammady H, Fedorowicz Z, et al. Rayyan-a web and mobile APP for systematic reviews. Syst Rev 2016;5:210.

40 Thompson JK, Heinberg LJ, Altabe M. Exacting beauty: theory, assessment, and treatment of body image disturbance. Washington, DC: American Psychological Association, 1999.

41 National Institute of Health. Quality assessment tool for observational cohort and cross-sectional studies, 2015. Available: https:// www.nhlbi.nih.gov/health-topics/study-quality-assessment-tools [Accessed 14 Aug 2020].

42 Beretvas SN. Meta-Analysis. In: Hancock GR, Mueller R, Stapleton $\mathrm{LM}$, eds, eds. The reviewer's guide to quantitative methods in the social sciences. New York, NY: Taylor \& Francis, 2010: 255-63.

43 Vrbik J. Population moments of sampling distributions. Comput Stat 2005;20:611-21.

44 Ulrich R, Wirtz M. On the correlation of a naturally and an artificially dichotomized variable. Br J Math Stat Psychol 2004;57:235-51.

45 Gelman A, Hwang J, Vehtari A. Understanding predictive information criteria for Bayesian models. Stat Comput 2014;24:997-1016.

46 Carpenter B, Gelman A, Hoffman MD, et al. Stan : A Probabilistic Programming Language. J Stat Softw 2017;76.

47 Stan Development Team, 2020the R interface to Stan. Available: http://mc-stan.org/

48 Gelman A. Prior distributions for variance parameters in hierarchical models. Bayesian Anal 2006;1:515-34.

49 Sterne JA, Egger M, Moher D. Addressing reporting biases. In: Higgins JPT, Green S, eds. Cochrane Handbook for systematic reviews of interventions. Chichester, UK: John Wiley \& Sons, 2008: 297-334.

50 Egger M, Smith GD, Schneider M, et al. Bias in meta-analysis detected by a simple, graphical test. BMJ 1997;315:629-34.

51 Sterne JAC, Sutton AJ, loannidis JPA, et al. Recommendations for examining and interpreting funnel plot asymmetry in meta-analyses of randomised controlled trials. BMJ 2011;343:d4002.

52 Duval S, Tweedie R. Trim and fill: a simple Funnel-Plot-Based method of testing and adjusting for publication bias in meta-analysis. Biometrics 2000;56:455-63.

53 Harring JR, McNeish DM, Hancock GR. Using phantom variables in structural equation modeling to assess model sensitivity to external misspecification. Psychol Methods 2017;22:616-31.

54 Guyatt G, Oxman AD, Akl EA, et al. Grade guidelines: 1. Introduction-GRADE evidence profiles and summary of findings tables. J Clin Epidemiol 2011;64:383-94.

55 Balshem $\mathrm{H}$, Helfand M, Schünemann $\mathrm{HJ}$, et al. Grade guidelines: 3. rating the quality of evidence. J Clin Epidemiol 2011;64:401-6.

56 Simoni JM, Amico KR, Pearson CR, et al. Strategies for promoting adherence to antiretroviral therapy: a review of the literature. Curr Infect Dis Rep 2008;10:515-21.

57 Santos CP, Felipe YX, Braga PE, et al. Self-perception of body changes in persons living with HIV/AIDS: prevalence and associated factors. AIDS 2005;19:S14-21. 